\title{
The role of gadoxetic acid as a paramagnetic contrast medium in the characterization and detection of focal liver lesions: a review*
}

O papel do ácido gadoxético como meio de contraste paramagnético na caracterização e detecção da lesão hepática focal: uma revisão

\section{Renata Lilian Bormann ${ }^{1}$, Eduardo Lima da Rocha ${ }^{1}$, Marcelo Longo Kierzenbaum ${ }^{1}$, Bruno Cheregati} Pedrassa $^{1}$, Lucas Rios Torres $^{2}$, Giuseppe D'lppolito ${ }^{3}$

Bormann RL, Rocha EL, Kierzenbaum ML, Pedrassa BC, Torres LR, D'Ippolito G. The role of gadoxetic acid as a paramagnetic contrast medium in the characterization and detection of focal liver lesions: a review. Radiol Bras. 2015 Jan/Fev;48(1):43-51.

Abstract Recent studies have demonstrated that the use of paramagnetic hepatobiliary contrast agents in the acquisition of magnetic resonance images remarkably improves the detection and differentiation of focal liver lesions, as compared with extracellular contrast agents. Paramagnetic hepatobiliary contrast agents initially show the perfusion of the lesions, as do extracellular agents, but delayed contrastenhanced images can demonstrate contrast uptake by functional hepatocytes, providing further information for a better characterization of the lesions. Additionally, this intrinsic characteristic increases the accuracy in the detection of hepatocellular carcinomas and metastases, particularly the small-sized ones. Recently, a hepatobiliary contrast agent called gadolinium ethoxybenzyl dimeglumine, that is simply known as gadoxetic acid, was approved by the National Health Surveillance Agency for use in humans. The authors present a literature review and a practical approach of magnetic resonance imaging utilizing gadoxetic acid as contrast agent, based on patients' images acquired during their initial experiment.

Keywords: Magnetic resonance imaging; Gadolinium; Liver; Contrast media.

Resu mo Estudos recentes têm demonstrado que a utilização dos agentes de contraste paramagnéticos hepatobiliares na obtenção das imagens de ressonância magnética hepática melhoram de maneira expressiva a detecção e diferenciação das lesões hepáticas focais, em comparação com a utilização de meios de contraste de ação apenas extracelular. 0 uso do meio de contraste hepatobiliar permite uma avaliação inicial da perfusão do tumor, da mesma forma que os agentes de contraste extracelulares, além de uma avaliação tardia da captação pelos hepatócitos funcionantes, fornecendo informações adicionais que permitem uma melhor caracterização das lesões. Além disso, a utilização do agente de contraste hepatobiliar pode aumentar a acurácia do método na detecção de metástases e do carcinoma hepatocelular, especialmente os de pequenas dimensões. Recentemente, foram aprovadas pela Agência Nacional de Vigilância Sanitária a utilização e a comercialização de um agente de contraste hepatobiliar, o gadolínio etoxibenzil dimeglumine, conhecido genericamente com ácido gadoxético. Revisamos a literatura atual e apresentamos uma abordagem prática da utilização da ressonância magnética com o ácido gadoxético utilizando exemplos de imagens de pacientes da nossa experiência inicial.

Unitermos: Ressonância magnética; Gadolínio; Fígado; Meios de contraste.

\section{INTRODUCTION}

Nowadays, in spite of the role played by ultrasonography (US) and computed tomography (CT) as the main tools in the screening of focal liver lesions (FLL), magnetic resonance imaging (MRI) plays a key role in the characterization of such lesions, thanks to technical advances of this

* Study developed at Department of Imaging Diagnosis - Escola Paulista de Medicina da Universidade Federal de São Paulo (EPM-Unifesp), São Paulo, SP, Brazil.

1. MDs, Fellows, Abdominal Imaging, Department of Imaging Diagnosis - Escola Paulista de Medicina da Universidade Federal de São Paulo (EPM-Unifesp), São Paulo, SP, Brazil.

2. MD, Master, Department of Imaging Diagnosis - Escola Paulista de Medicina da Universidade Federal de São Paulo (EPM-Unifesp), São Paulo, SP, Brazil.

3. Associate Professor, Department of Imaging Diagnosis - Escola Paulista de Medicina da Universidade Federal de São Paulo (EPM-Unifesp), São Paulo, SP, Brazil. method with the development of fast sequences and new techniques such as diffusion-weighted imaging and, most recently, the introduction of hepatospecific contrast media ${ }^{(1-4)}$.

In MRI, contrast agents have demonstrated their usefulness in the imaging of a variety of organs, for improved detection and characterization of several lesions and functional abnormalities, since the study performed with the utilization of contrast medium adds morphological and functional information to non-contrast-enhanced imaging studies $^{(1,5,6)}$. Currently, a variety of contrast agents have been utilized for liver MRI studies, most of them based on gadolinium ion chelates which have been utilized from late

Mailing Address: Dr. Giuseppe D'Ippolito. Departamento de Diagnóstico por Imagem - EPM-Unifesp. Rua Napoleão de Barros, 800, Vila Clementino. São Paulo, SP, Brazil, 04024-012. E-mail: giuseppe_dr@uol.com.br.

Received May 7, 2013. Accepted after revision February 6, 2014. 
$1980 \mathrm{~s}^{(7,8)}$. The types of gadolinium-based contrast media currently in the marketplace may be divided into two categories - nonspecific extracellular and specific intracellular agents - with the main difference between the two types being the chelating molecule that carries the gadolinium ${ }^{(8)}$. The nonspecific extracellular gadolinium was the first category of MRI contrast agents approved for clinical use, with an excellent safety track record for patients with normal renal function ${ }^{(1)}$. More recently, specific intracellular contrast agents have been developed for liver MRI studies, in order to overcome the limitations of extracellular gadolinium chelates, thus being called hepatospecific contrast agents ${ }^{(7)}$. The two main classes of hepatospecific contrast agents are superparamagnetic iron oxide, which presents selective uptake by the reticuloendothelial system, particularly by liver and spleen, and hepatobiliary contrast agents, which are uptaken by the hepatocytes and are excreted by the renal and biliary tracts ${ }^{(7,9,10)}$. Thus, the hepatobiliary contrast agents provide, initially, perfusional data similar to those from nonspecific extracellular gadolinium (with renal excretion), and later, hepatocyte-selective data (with biliary excretion), thus allowing for the differentiation between lesions containing hepatocytes and lesions without functional hepatocytes $^{(9,10)}$

In Brazil, Agência Nacional de Vigilância Sanitária (National Health Surveillance Agency) has recently approved the commercialization and utilization of a hepatobiliary contrast agent, gadolinium ethoxybenzyl dimeglumine (Gd-EOBDTPA, gadoxetate disodium, gadoxetic acid disodium, Primovist $\left.{ }^{\circledR}\right)$, generally known as gadoxetic acid. Such a contrast agent, already in use in the United States of America $\left(\right.$ Eovist $\left.^{\circledR}\right)$, Europe (Primovist ${ }^{\circledR}$ ) and Asia, has demonstrated to be useful to improve the detection and characterization of FLLs ${ }^{(4,10-15)}$.

Between October 2012 and February 2013, the authors had the opportunity to perform twenty hepatobiliary MRI studies in their service with the utilization of gadoxetic acid. Some of those cases were selected as being illustrative and useful for the understanding on the behavior, utilization and value of the gadoxetic acid in the investigation of FLLs.

\section{PHARMACOLOGICAL CHARACTERISTICS}

Gadoxetic acid is a paramagnetic contrast medium utilized in MRI scans, whose enhancement effect is mediated by gadoxetate, an ionic complex formed by gadolinium and the ethoxybenzyl diethylenetriamine pentaacetic acid ligand (EOB-DTPA). Because of the lipophilic property of the ethoxybenzyl component, the gadoxetate disodium provides a biphasic or two-compartmental action: after intravenous Gd-EOB-DTPA injection, the agent distributed within the vessels and in the extracellular spaces (vascular/interstitial space) during the dynamic enhancement phases (arterial, portal and equilibrium or transition phases), and later undergoes progressive uptake by normal functional hepatocytes, being completely eliminated by the renal and hepatobiliary tracts, in similar amounts ( $50 \%$ each), as the functioning of such organs is normal ${ }^{(11,12,16,17)}$. Because of this action profile, the gadoxetic acid is considered as being a mixed-action contrast agent: extracellular and hepatobiliary.

Gadoxetic acid is an ionic contrast medium with a linear molecular structure. The uptake by the hepatocytes occurs mainly by means of a transportation protein present in the sinusoidal membrane (OATP1B1 and B3), and later the biliary excretion is obtained by means of proteins located in the canalicular membrane (MRP2). On account of such characteristics, the Gd-EOB-DTPA behaves similarly to the nonspecific (or extracellular) gadolinium chelates during the dynamic phases and provides additional data during the hepatobiliary excretion. In that phase, the normal liver parenchyma with functional hepatocytes uptakes or concentrates the contrast medium; the lesions without normal functional hepatocytes do not uptake the contrast medium (for example, metastases), thus allowing for a better evaluation and characterization of the FLL ${ }^{(11,12)}$.

The Gd-EOB-DTPA has a high capability of binding with proteins that significantly increase the gadoxetic acid $\mathrm{T} 1$ relaxivity, which provides good enhancement effect of the vessels and the liver, allowing for a reduction of dose as compared with other nonspecific gadolinium-based contrast media. However, the resulting effect of T1 shortening for dynamic images is more subtle, in particular for vascular enhancement, as compared with nonspecific gadolinium chelates, a fact that makes it not ideal for angiographic studies $^{(11,12,18)}$.

The gadoxetic acid must be applied by means of intravenous (either arterial or venous) bolus injection, at a dose of $0.025 \mathrm{mmol} / \mathrm{kg}$ of body weight $(0.1 \mathrm{~mL} / \mathrm{kg})$, which corresponds to one-half of the dose for nonspecific extravascular gadolinium usually utilized in abdominal studies ${ }^{(12)}$.

The gadoxetic acid is not metabolized and, in healthy patients, is also eliminated through the renal and hepatobiliary tracts. In patients presenting with terminal renal dysfunction, it can be eliminated by means of dialysis. Although the systemic body exposure to gadolinium is low, considering the small dose and double elimination pathway (renal and hepatobiliary tracts), there is a possibility of occurrence of systemic nephrogenic fibrosis. Therefore, gadoxetic acid can only be utilized in patients with severe renal dysfunction, after a careful risk/benefit evaluation. Its half-life is approximately 2 hours, the peak of accumulation in the hepatocyte occurs between 20 and 40 minutes, and the beginning of hepatocytic concentration and biliary excretion occur, respectively, after three and ten minutes. The compound does not cross the intact hematoencephalic barrier and diffuses through the placental barrier only in a small concentration $^{(16,17)}$

Similarly to other intravenously injected contrast media, Gd-EOB-DTPA may be associated with anaphylactoid/ hypersensitivity reactions or to other idiosyncratic reactions characterized by cardiovascular, respiratory or cutaneous 
manifestations, occasionally causing severe reactions, including shock. Gadoxetic acid is well tolerated, with side effects similar to those reported in the utilization of nonspecific gadolinium chelate, namely: nausea $(1 \%)$, headache $(0.9 \%)$, lumbar pain $(0.5 \%)$, vertigo $(0.4 \%)$, vasodilation $(0.6 \%)$, dysgeusia and pain at the injection site $\mathrm{e}^{(7,11,13,15-17,19)}$. In the authors' experience, no adverse side effects or limitations to the use of ethoxybenzyl were observed.

There are no data available in the literature about exposure to gadoxetic acid during pregnancy. In clinical doses, no effect to the infant is expected, and it may be utilized during breastfeeding period. Dose adjustments are not required in elderly patients ( $>65$ years) as well as in patients with hepatic dysfunction and renal dysfunction. Increased bilirubin $(>3 \mathrm{mg} / \mathrm{dl})$ or ferritin levels might reduce the enhancement effect in the liver ${ }^{(16,17)}$.

\section{IMAGING PROTOCOL}

The recommended imaging protocol includes non-contrast-enhanced sequences, T1-weighted gradient-echo inphase and out-of-phase sequences, fast $\mathrm{T} 2$-weighted sequences with fat saturation and a phase with intravenous contrast bolus injection utilizing the dose of $0.1 \mathrm{~mL} / \mathrm{kg}$ of Gd-EOB-DTPA (equivalent to $0.025 \mathrm{mmol} / \mathrm{kg}$ ), either manual or by means of an automatic infusion pump, at a rate of $1 \mathrm{~mL} / \mathrm{s}$, followed by a $20 \mathrm{~mL}$ saline solution flush at the same infusion rate ${ }^{(20)}$. After the contrast agent injection, a T1-weighted gradient echo sequence with fat saturation is obtained, in the arterial phase (15 to 20 seconds after initiating the intravenous injection), portal phase (50 to 60 seconds), equilibrium or transition phase ( 120 seconds) and in the hepatobiliary phase (10 and 20 minutes after initiating the intravenous injection $)^{(4,12)}$. The total scan time is approximately 30 to 40 minutes, but such time may be reduced by performing the $\mathrm{T} 2$-weighted sequence and the diffusion sequence between the equilibrium phase and the hepatobiliary phase ${ }^{(4,21)}$. In liver MRI, the diffusion sequence is generally added to the routine protocols, and is usually performed before the intravenous contrast injection. However, it has been demonstrated that the diffusion sequence may be performed after the gadoxetic acid injection, before the images acquisition in the hepatobiliary phase, reducing the scan time, without compromising the values of the apparent diffusion coefficient and the contrast/ noise ratio of the lesion ${ }^{(22)}$. Additionally, several studies have demonstrated that, in non-cirrhotic patients, the hepatobiliary phase may be performed earlier, i.e., 10 minutes after the intravenous contrast injection, without affecting MRI results $^{(4,21)}$. At the authors' service, the MRI apparatuses operate at $1.5 \mathrm{~T}$, with synergy coils (Magnetom Sonata ${ }^{\circledR}$; Siemens, Erlangen, Germany, and Gyroscan Intera ${ }^{\circledR}$; Phillips Medical Systems, Best, The Netherlands).

\section{INDICATIONS}

The clinical application of this new contrast media must be understood as a new tool to solve problems with patients with FLL with atypical characteristics, in cases where there is suspicion of either primary or secondary liver tumors not clearly identified with other methods, and to complement the data provided by the utilization of nonspecific extracellular gadolinium chelates or by contrast-enhanced $\mathrm{CT}^{(1)}$.

One of the main indications for the utilization of gadoxetic acid is the differentiation between hepatocellular and non-hepatocellular FLLs. In that sense, it may be utilized to differentiate lesions containing biliary ducts, such as dysplastic nodules in cirrhotic patients and focal nodular hyperplasia (FNH) in non-cirrhotic patients, from non-hepatocellular lesions, such as hepatocellular carcinoma (HCC), adenoma, metastasis and hemangioma. Table 1 shows the main indications for the utilization of gadoxetic acid in the evaluation of the liver by means of MRI, and adopted e by the authors in their studied cases.

Table 1-Main indications for the utilization of gadoxetic acid in liver MRI scans.

Differentiation between FLL of hepatocellular origin and non-hepatocellular lesions $^{(23)}$

Differentiation between FNH and adenoma ${ }^{(\mathbf{1 , 4 , 2 4 , 2 5 )}}$

Detection of small HCC $(<2.0 \mathrm{~cm})^{(26-29)}$

Pre-transplant evaluation in HCC patients ${ }^{(4)}$

Detection of liver metastasis ${ }^{(\mathbf{1 1}, \mathbf{1 3}, \mathbf{3 0})}$

Note: The numbers between parentheses correspond to the bibliographic references for each indication.

\section{FNH versus adenoma}

FNH and adenoma are, respectively, the second and third most common benign liver tumors ${ }^{(25)}$ and affect patients with similar epidemiologic profiles ${ }^{(26)}$. In spite of the fact that both lesions are considered to be benign, their differentiation is important because of possible complications associated with adenomas, such as risk for bleeding and malignant transformation into HCC, which require appropriate clinical management ${ }^{(25)}$. Sometimes, the imaging findings of these tumors overlap, making their differentiation more difficult. In such cases, gadoxetic acid may be useful to differentiate such entities ${ }^{(1,25)}$.

FNH is defined as a frequently single, well circumscribed liver lesion, characterized by a fibrotic central scar, surrounded by hyperplastic hepatocyte agglomerates and small biliary ducts, in a liver with normal histological appearance $^{(31)}$. Due to the fact that this lesion presents with immature bile canaliculi which do not communicate with biliary ducts of larger caliper, there is a greater uptake of the hepatospecific contrast medium by the lesion than by the adjacent normal liver parenchyma. Additionally, at the images acquired with Gd-EOB-DTPA, an either homogeneous or heterogeneous enhancement of the lesion at the hepatocellular phase can be observed, depending on the amount of fibrosis and its distribution in the lesion (also known as "central scar"). Thus, the FNH becomes iso- or hyperintense in relation to the normal liver, allowing for its differentiation from other lesions, even in cases where heterogeneous enhancement is observed (Figure 1) $)^{(10,24,31)}$. 

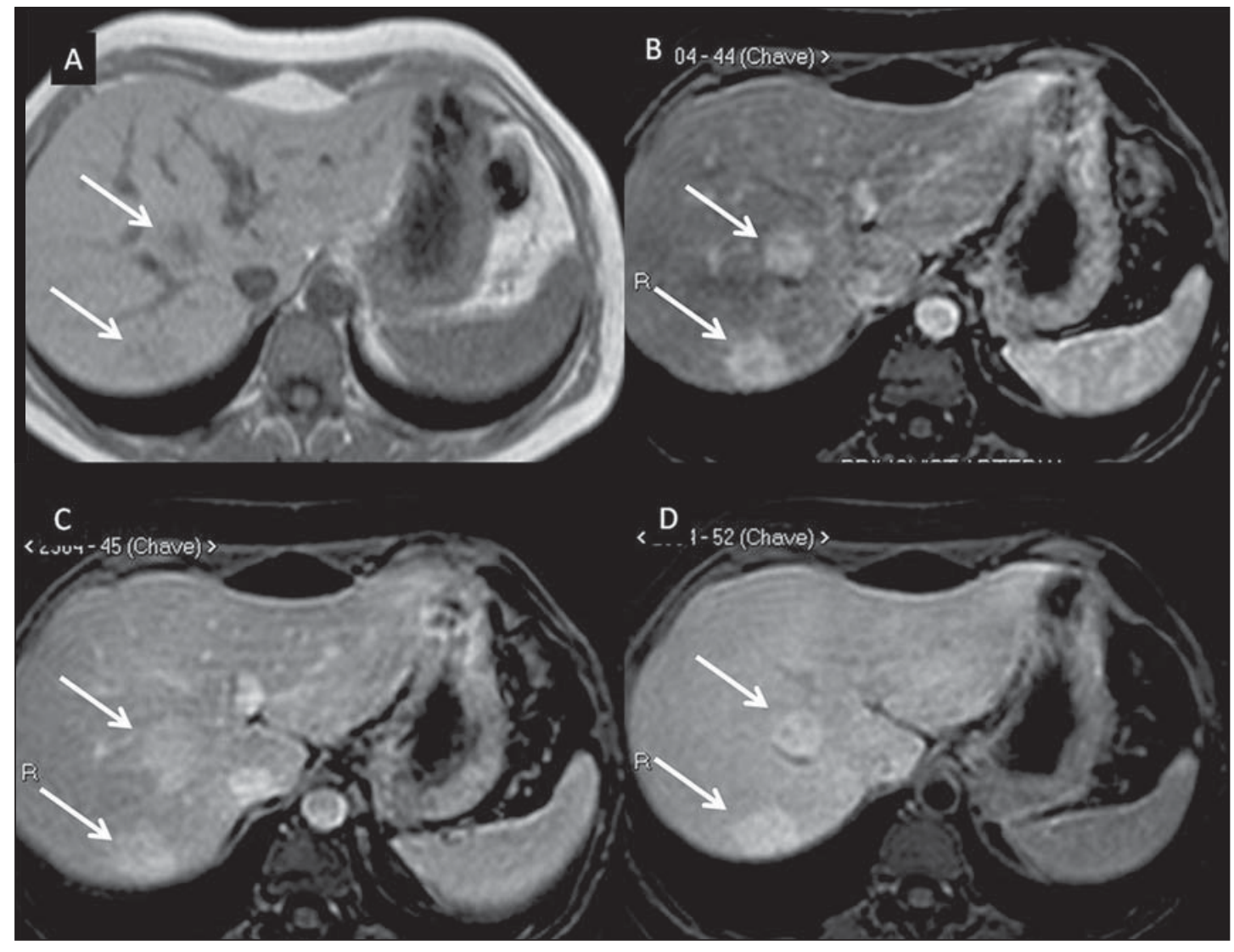

Figure 1. FNH (diagnosis based on histological analysis after percutaneous biopsy). The two nodules in the right hepatic lobe (arrows) present with hyposignal on T1-weighted image $(\mathbf{A})$, early and intense contrast en hancement in the arterial phase $(\mathbf{B})$, persisting in the portal phase $(\mathbf{C})$ and in the hepatobiliary phase performed 10 minutes after initiation of the intravenous gadoxetic acid injection (D).

Histologically, hepatic adenomas consist of well differentiated hepatocyte cords, with absence biliary ducts or portal tracts ${ }^{(10,32)}$. At images with Gd-EOB-DTPA, adenomas typically present contrast uptake in up to $100 \%$ of the cases, but lower than the uptake by the parenchyma in the hepatobiliary phase, due to the absent or quite reduced hepatocellular uptake of gadoxetic acid by the lesion (Figure 2). On the other hand, in up to $10 \%$ of cases, a similar or greater uptake can occur in relation to the parenchyma in the hepatobiliary phase $\mathrm{e}^{(33)}$, or peripheral ring-shaped enhancement, since some hepatocytes maintain the capacity of absorption and excretion in the hepatocellular phase. This may represent a confusing factor in the diagnosis of adenoma, making it similar to $\mathrm{FNH}^{(10,23,33)}$.

The presence of fat in the adenoma allows an easy differentiation from FNH, as the latter rarely contains fat ${ }^{(34)}$. Recently, however, three subtypes of adenomas were described on the basis of histological differences, as follows: steatotic, inflammatory and activated beta-catenin. Adenomas of the inflammatory subtype correspond to approximately $40 \%$ of such tumors and present a low fat content ${ }^{(35)}$. At nonspecific contrast-enhanced MRI, adenomas present marked arterial enhancement keeping an iso- or subtle hypersignal in relation to the parenchyma at the delayed phases, with difficult differentiation from FNHs. In such cases of diagnostic doubt between FNH and adenoma, gadoxetic acid may be particularly useful ${ }^{(36)}$. On the other hand, adenomas of activated beta-catenin subtype may present signal hyperintensity in the hepatobiliary phase, after the hepatospecific contrast injection, remaining as a source of diagnostic doubt.

\section{Hepatic nodules in cirrhotic patients}

HCC is the most common malignant primary hepatic neoplasm, generally occurring as a complication of hepatic cirrhosis, particularly that caused by $\mathrm{B}$ and $\mathrm{C}$ viruses ${ }^{(10,32)}$. HCC is the main cause of deaths in cirrhotic patients, and for this reason an early and accurate diagnosis is very important for an appropriate treatment and management of such patients ${ }^{(29)}$. Nodular lesions in a cirrhotic liver can be divided into two major groups: a) regenerative and dysplastic nodules; b) neoplastic nodules ${ }^{(32)}$. However, the correct imaging characterization of these lesions still remains a challenge, as frequently pre-neoplastic hepatocellular lesions as well as dysplastic nodules mimic small HCCs well-differentiated ${ }^{(37)}$.

The diagnosis of non-invasive HCC has been made according to the "Barcelona Criteria" which adopt imaging methods, particularly CT and MRI with intravenous contrast agent, to characterize as HCC a focal lesion in a cirrhotic liver; HCCs are those nodules $\geq 2.0 \mathrm{~cm}$ in diameter, hypervascular in the arterial phase (wash-in) and wash-out in delayed phases, at contrast-enhanced axial images (either CT or MRI $)^{(38)}$. In cases where a liver lesion is between 1.0 and $2.0 \mathrm{~cm}$ in diameter, the guidelines by American Association for Studies of Liver Diseases recommend that the diagnosis of HCC be based on two dynamic imaging studies with typical findings ${ }^{(39)}$. However, such typical imaging 


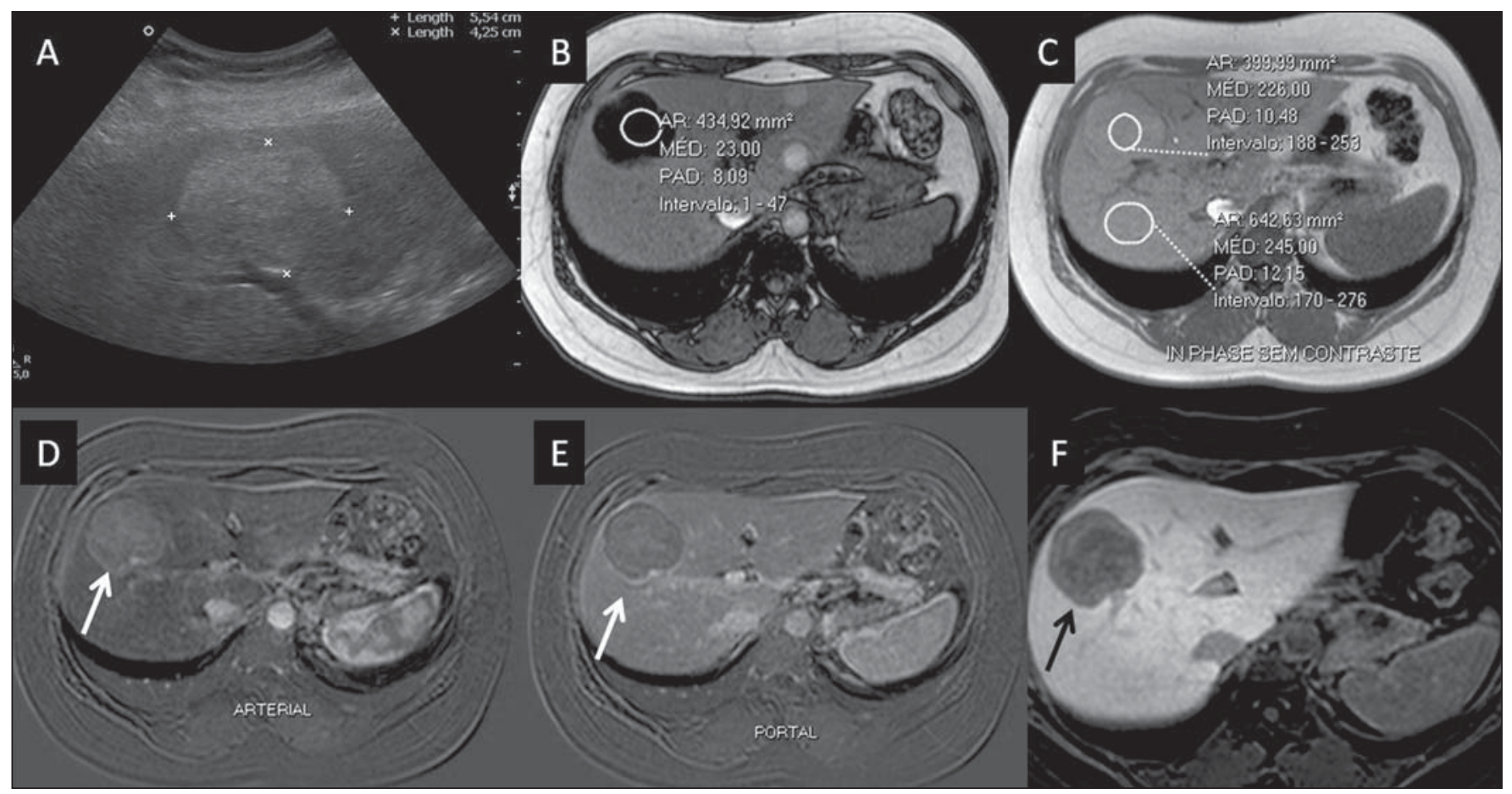

Figure 2. Hepatic adenoma (diagnosis based on histological analysis after percutaneous biopsy). US demonstrates the presence of a hyperechogenic and homogeneous mass of $5.0 \mathrm{~cm}$ in diameter (marked with a caliper) (A). MRI out of phase image (B) demonstrates signal intensity drop as compared with the in-phase image (C), indicating the presence of intracellular fat component. After intravenous gadoxetic acid injection, with subtraction technique, a lesion (arrows) is observed with subtle contrast enhancement in the arterial phase (D), imperceptible in the portal phase (E) and without contrast uptake in the hepatobiliary phase (F).

findings are not frequently observed in cases of small HCCs $(<2.0 \mathrm{~cm})$, particularly in cases of well-differentiated early HCCs, requiring liver biopsy for diagnosis and close follow-up ${ }^{(29)}$.

In cirrhotic patients, the gadoxetic acid may be useful in the identification of small HCCs and in the correct characterization of lesions presenting atypical behavior at $\mathrm{T} 1$ - and T2-weighted images, with a nonspecific enhancement pattern $^{(4,29)}$. In the hepatobiliary phase after gadoxetic acid injection, regenerative nodules show up iso- or slightly hyperintense in relation to the surrounding liver parenchyma, as they are composed of functioning hepatocytes, and are many times identified by the presence of a thin pseudocapsule with hyposignal resulting from the surrounding fibrous matrix ${ }^{(37)}$. On the contrary, HCCs present hyposignal in relation to the adjacent liver parenchyma in the hepatobiliary phase, particularly in cases of moderately or poorly differentiated lesions $^{(29)}$ (Figure 3). Dysplastic nodules represent a common finding in the cirrhotic liver, and may be classified into lowand high-grade dysplastic nodules, on the basis of the number and type of cellular atypias. High-grade dysplastic nodules are considered as being pre-malignant lesions. Even with the utilization of gadoxetic acid, the differentiation between dysplastic nodule and well-differentiated HCC still represents a diagnostic challenge, as in some cases neoplastic cells of well-differentiated HCC may present with preserved hepatocellular function, being capable of absorption and metabolization of the contrast medium and, so the malignant nodule can be seen either as iso- or even hyperintense in the hepato- biliary phase, simulating a regenerative or a dysplastic nod$u_{l}^{(29,37)}$. On the other hand, some dysplastic nodules are seen as hypointense in the hepatobiliary phase, mimicking a HCC $^{(29)}$ (Figure 4).

Recent studies have demonstrated that the Gd-EOBDTPA uptake by some HCCs is related to the expression of transporting proteins - OATP1B3 - in the hepatocytes membrane of these lesions ${ }^{(40)}$. However, further studies are necessary to confirm such a theory.

Although multidetector CT (MDCT) has achieved a high standard in the detection of HCC, due to the possibility of multiphase scans and a set of high resolution data, MRI is considered the best noninvasive imaging method for detecting HCC and for characterizing nodules in cirrhotic patients, because the multiple evaluated parameters, and especially due to the possibility of utilization of hepatospecific contrast agents ${ }^{(41)}$. Several studies have demonstrated an increase in the rate of detection of HCC by MRI with gadoxetic acid, as compared with MDCT, especially in cases of lesions $<1.5 \mathrm{~cm}^{(42-44)}$. In 2009, Kim et al. demonstrated greater accuracy in the diagnosis of HCC by MRI with GdEOB-DTPA, with $91.45 \%$ sensitivity in the gadoxetic acid group, versus $71.6 \%$ in the MDCT group, with a $24.7 \%$ higher percentage in the detection of small HCCs $(<1.5$ $\mathrm{cm})$. Other investigators have demonstrated that the combination of dynamic study with the hepatocyte phase at gadoxetic acid-enhanced MRI had a better diagnostic performance than the dynamic study alone in the characterization of focal lesions in cirrhotic livers ${ }^{(45,46)}$. 

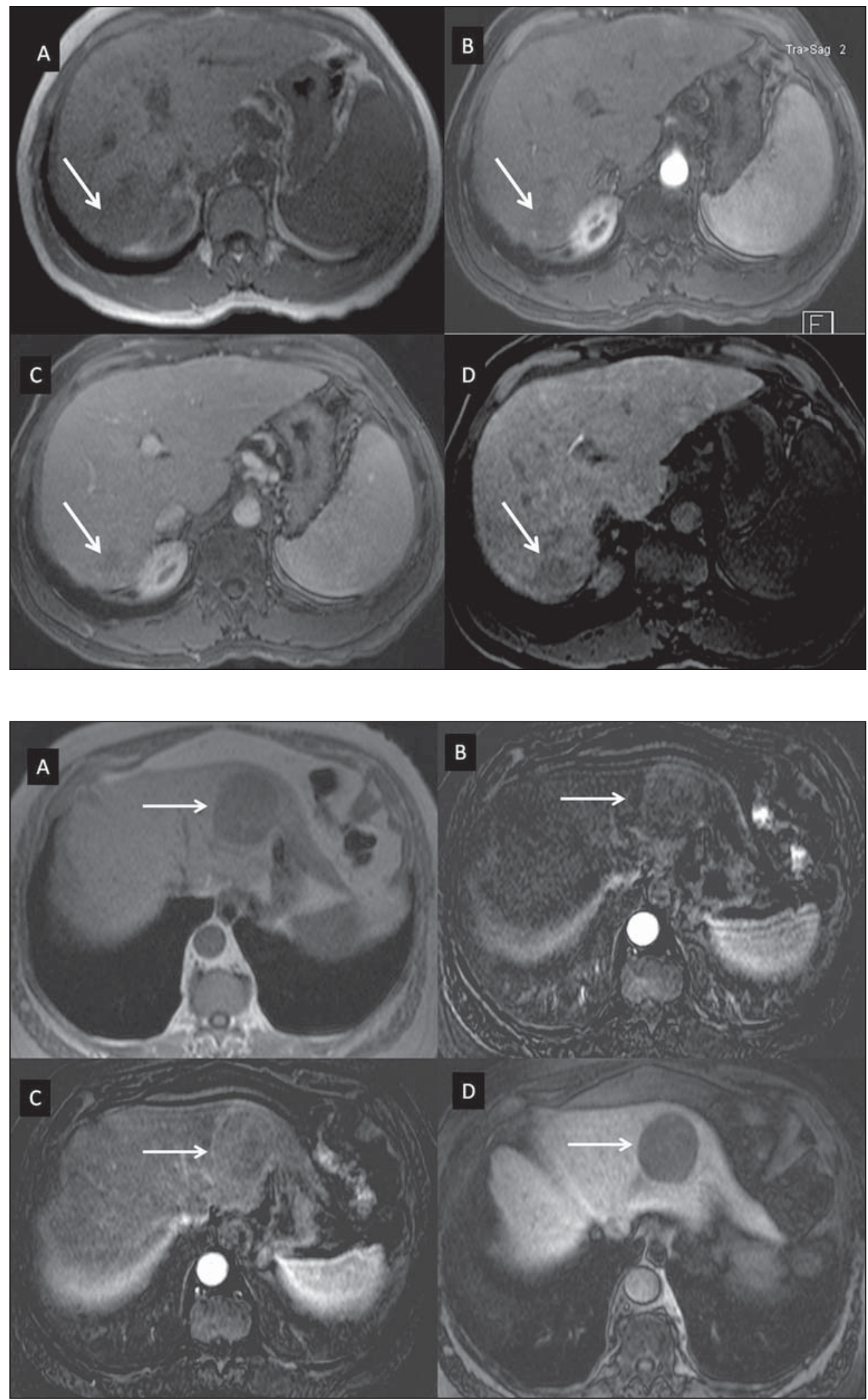

Figure 3. Infiltrative and undifferentiated HCC (confirmed by percutaneous biopsy). At T1-weighted images before intravenous gadoxetic acid injection (A) and obtained in the arterial and portal contrast phases (B,C), the lesion is poorly defined as compared with the image obtained in the hepatobiliary phase (arrows), acquired 20 minutes after the utilization of the contrast medium (D). Notice that, in this phase, contrast uptake by the lesion is lower in relation to the hepatic parenchyma, indicating the absence of normal functional hepatocytes.

Figure 4. Dysplastic nodule versus well differentiated HCC. At T1-weighted image (A) a bulky hypointense, homogeneous and well-defined mass is observed in the left liver lobe (arrows). In the arterial (B) and portal (C) phases, images acquired with the subtraction technique to potentialize the identification of the lesion enhancement, demonstrate an early, subtle enhancement, without unequivocal wash-out. In the hepatobiliary phase (D), obtained $20 \mathrm{~min}$ utes after the intravenous gadoxetic acid injection, no uptake by the lesion was observed. The histological analysis of the biopsy specimen could not differentiate between a high-grade dysplastic nodule and a well-differentiated HCC.

\section{Liver metastasis}

Another use for gadoxetic acid contrast media in the study of the liver is the detection of liver metastases, particularly in the follow-up of patients with colorectal carcinoma ${ }^{(4,47)}$. Hepatic metastasis is the most frequent malig- nant liver lesion. The correct diagnosis is fundamental for the definition of the therapeutic approach as well as to establish the prognosis, hence the relevance of the differentiation between such lesions and other benign liver nodules in cancer patients $^{(32)}$. In the gadoxetic acid-enhanced hepato- 
biliary phase, both hypovascular and hypervascular liver metastases are hypointense in relation to the adjacent parenchyma due to the absence of functional hepatocytes in such lesions ${ }^{(30)}$. In that phase, the lesion washout in association with the enhancement of the surrounding healthy parenchyma improves the liver-tumor contrast, increasing the conspicuity of the lesion. This allows for a significantly higher rate of lesions detection, especially for those $<1.0 \mathrm{~cm}$ in diameter, a fact that may impact the therapeutic planning as well as the surgical approach ${ }^{(15,19,30)}$ (Figure 5). In a prospective study, Hammerstingl et al. ${ }^{(13)}$ have demonstrated that gadoxetic acid-enhanced MRI was superior as compared with CT in the evaluation of FLL, considering detection, localization, delimitation and management of patients, leading to changes in the therapeutic approach in $14.5 \%$ of the patients, allowing for better preoperative planning in cases of liver resection. Another study has also demonstrated a higher rate of detection of liver metastases with the utilization of gadoxetic acid-enhanced MRI, both for lesions smaller and larger than $1.0 \mathrm{~cm}^{(19)}$.

\section{Other liver lesions}

For the remaining liver nodules such as the hemangioma, cholangiocarcinoma and other benign lesions (for example: abscesses and hydatid cysts), gadoxetic acid does not seem to be a precise indication, considering that in such cases there is no enhancement of the lesions in the hepatobiliary phase, as such nodules do not have functional hepatocytes. An exception would be benign lesions of biliary ducts, such as Caroli disease, where enhancement of cystic lesions in the hepatobiliary phase is observed because of their communication with the biliary tree ${ }^{(48)}$.
The enhancement of hemangiomas at Gd-EOB-DTPAenhanced MRI presents some particularities. The lesion tends to follow the signal from the blood in the abdominal vessels in the extracellular phase. On the other hand, contrary to what occurs as the extracellular contrast medium is utilized, in the hepatobiliary phase, the hemangioma does not present progressive or persistent, and higher or equal enhancement in relation to the liver parenchyma, but rather hyposignal in relation to the liver, because of the absence of hepatocytes, in contrast with the normal adjacent parenchyma, which presents intense Gd-EOB-DTPA uptake in this phase. Such a phenomenon is called liver/lesion enhancement gradient inversion $^{(4,10,14)}$ (Figure 6).

\section{CONCLUSION}

The present study was aimed at reviewing the MRI evaluation of liver nodules with the utilization of hepatospecific contrast medium, considering the recent availability of such contrast agent in the market. In their initial experiment, substantiated by the literature review ${ }^{(1-3,11,13)}$, the authors could identify different enhancement patterns in lesions studied with gadoxetic acid as a function of their etiology, divided into two groups as follows: a group including lesions with functioning hepatocytes, such as FNH and dysplastic nodules, and another group comprising all the remaining liver lesions which do not contain functioning hepatocytes (for example: HCC, adenomas and metastasis). The authors have observed that the utilization of ethoxybenzyl is more useful in the differentiation between FNH and adenoma, in the differentiation between dysplastic nodules and HCC in cirrhotic liver, in the detection of small HCCs $(<2.0 \mathrm{~cm}$ diameter) and tiny metastasis, where the hepatospecific con-
Figure 5. Liver metastasis from breast neoplasm (diagnosis based on periodic follow-up). The CT images acquired in the portal contrast phase (A) and the MRI T2-weighted sequence (B) allow for the identification of a single liver nodule in the VII/VIII segment (arrows on $\mathbf{A}$ and B). Gadoxetic acid-enhanced MRI portal phase (C) and hepatobiliary phase (D) allow for the identification of another small nodule (arrow on D), more clearly characterized in the delayed phase. Two cysts are also observed in the left lobe (arrowhead on B).

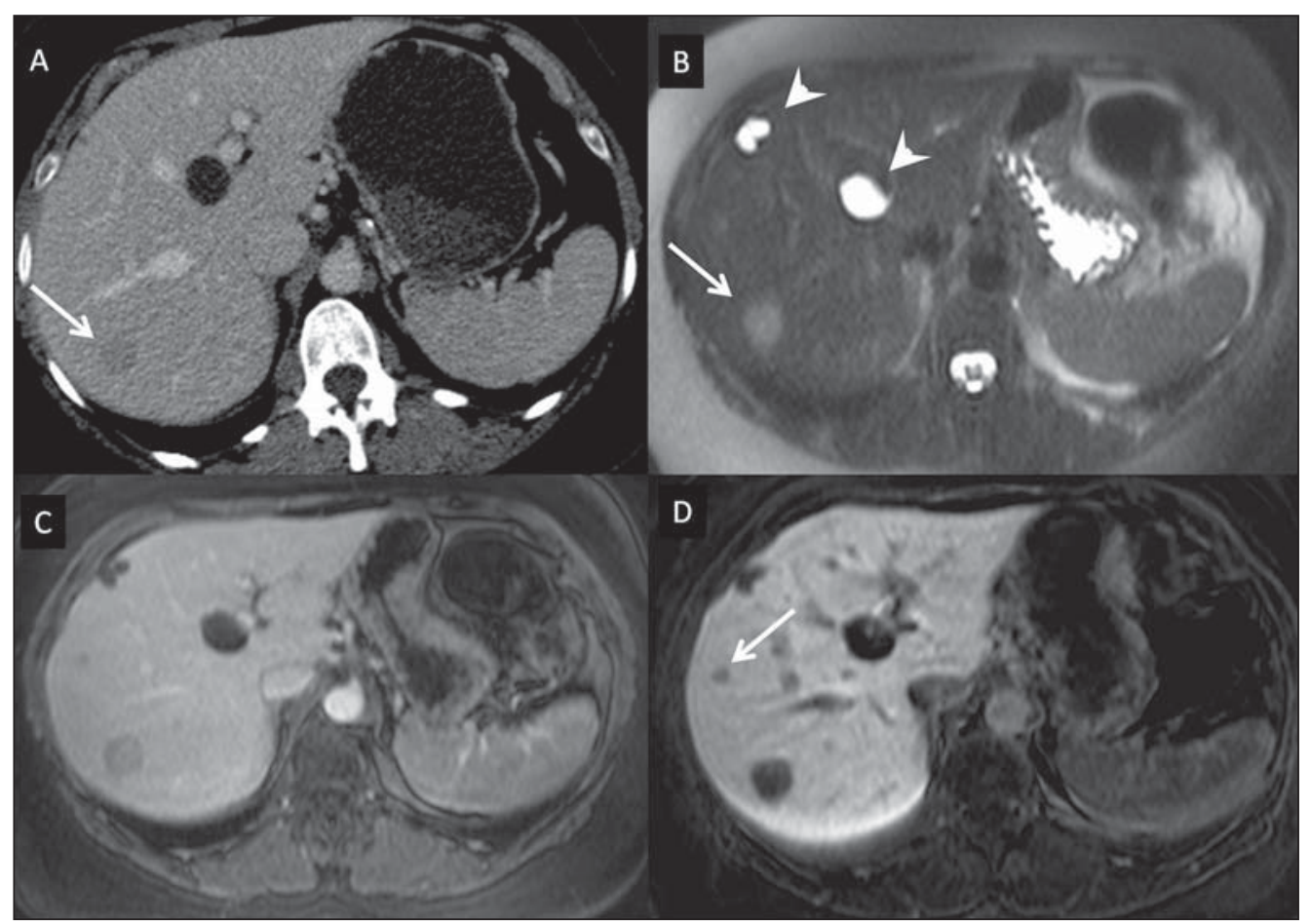




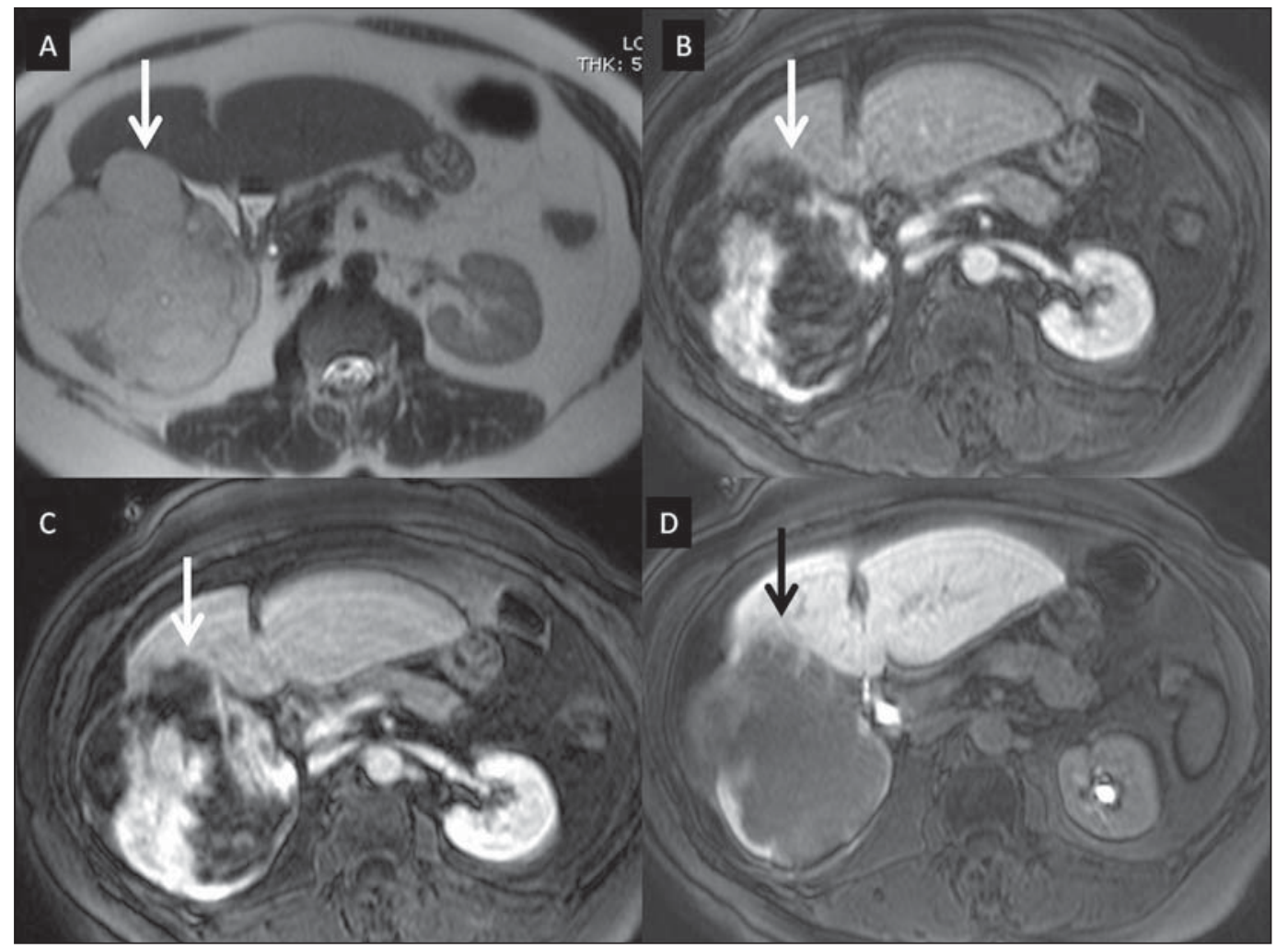

Figure 6. Hepatic hemangioma (diagnosis based on imaging findings). Presence of a lesion in the right liver lobe (arrows). MRI T2 weighted MRI sequence (A) demonstrates the typical hypersignal from the hemangioma, as well progressive, discontinuous and centripetal enhancement in the portal (B) and equilibrium (C) phases. In the hepatobiliary phase obtained 10 minutes after the intravenous gadoxetic acid injection, the liver/ lesion enhancement gradient inversion is observed, caused by the greater contrast uptake by the parenchyma, as compared with the low contrast uptake by the hemangioma, due to the absence of functioning hepatocytes (D)

trast agents demonstrate greater sensitivity and specificity than extracellular agents.

For such reasons, MRI with the utilization of ethoxybenzyl is currently considered as the best imaging modality for the investigation of FLLs ${ }^{(2)}$; but, because of its higher cost, such type of contrast agent should not be utilized on a routine basis, remaining reserved for selected cases where its usefulness has been proven, as in the case of the above mentioned indications.

\section{REFERENCES}

1. Ba-Ssalamah A, Uffmann M, Saini S, et al. Clinical value of MRI liver-specific contrast agents: a tailored examination for a confident non-invasive diagnosis of focal liver lesions. Eur Radiol. 2009; 19:342-57.

2. Holzapfel K, Eiber MJ, Fingerle AA, et al. Detection, classification, and characterization of focal liver lesions: value of diffusion-weighted MR imaging, gadoxetic acid-enhanced MR imaging and the combination of both methods. Abdom Imaging. 2012;37:74-82

3. Bartolozzi C. MR of the liver: from breakthrough to clinical application. Abdom Imaging. 2012;37:154.

4. Tanimoto A, Lee JM, Murakami T, et al. Consensus report of the 2nd International Forum for Liver MRI. Eur Radiol. 2009;19 Suppl 5:S975-89.

5. Bellin MF, Webb JA, Van Der Molen AJ, et al. Safety of MR liver specific contrast media. Eur Radiol. 2005;15:1607-14.

6. Bellin MF, Vasile M, Morel-Precetti S. Currently used non-specific extracellular MR contrast media. Eur Radiol. 2003;13:2688-98.

7. Reimer P, Schneider G, Schima W. Hepatobiliary contrast agents for contrast-enhanced MRI of the liver: properties, clinical development and applications. Eur Radiol. 2004;14:559-78.

8. Elias Jr J, Santos AC, Koenigkam-Santos M, et al. Complications from the use of intravenous gadolinium-based contrast agents for magnetic resonance imaging. Radiol Bras. 2008;41:263-7.
9. Semelka RC, Helmberger TK. Contrast agents for MR imaging of the liver. Radiology. 2001;218:27-38.

10. Campos JT, Sirlin CB, Choi JY. Focal hepatic lesions in Gd-EOBDTPA enhanced MRI: the atlas. Insights Imaging. 2012;3:45174.

11. Van Beers BE, Pastor CM, Hussain HK. Primovist, Eovist: what to expect? J Hepatol. 2012;57:421-9.

12. Seale MK, Catalano OA, Saini S, et al. Hepatobiliary-specific MR contrast agents: role in imaging the liver and biliary tree. Radiographics. 2009;29:1725-48.

13. Hammerstingl R, Huppertz A, Breuer J, et al. Diagnostic efficacy of gadoxetic acid (Primovist)-enhanced MRI and spiral CT for a therapeutic strategy: comparison with intraoperative and histopathologic findings in focal liver lesions. Eur Radiol. 2008;18:457-67.

14. Reimer P, Rummeny EJ, Daldrup HE, et al. Enhancement characteristics of liver metastases, hepatocellular carcinomas, and hemangiomas with Gd-EOB-DTPA: preliminary results with dynamic MR imaging. Eur Radiol. 1997;7:275-80.

15. Huppertz A, Balzer T, Blakeborough A, et al. Improved detection of focal liver lesions at MR imaging: multicenter comparison of gadoxetic acid-enhanced MR images with intraoperative findings. Radiology. 2004;230:266-75.

16. Primovist. Bula. Berlim: Bayer Pharma AG; 2011.

17. Tanimoto A, Kadoya M, Kawamura Y, et al. Safety and efficacy of a novel hepatobiliary MR contrast agent, Gd-DTPA-DeA: results of phase I and phase II clinical trials. J Magn Reson Imaging. 2006;23:499-508.

18. Tamada T, Ito K, Sone T, et al. Dynamic contrast-enhanced magnetic resonance imaging of abdominal solid organ and major vessel: comparison of enhancement effect between Gd-EOB-DTPA and Gd-DTPA. J Magn Reson Imaging. 2009;29:636-40.

19. Bluemke DA, Sahani D, Amendola M, et al. Efficacy and safety of MR imaging with liver-specific contrast agent: U.S. multicenter phase III study. Radiology. 2005;237:89-98.

20. Zech CJ, Vos B, Nordell A, et al. Vascular enhancement in early 
dynamic liver MR imaging in an animal model: comparison of two injection regimen and two different doses Gd-EOB-DTPA (gadoxetic acid) with standard Gd-DTPA. Invest Radiol. 2009;44:305-10.

21. Motosugi U, Ichikawa T, Tominaga L, et al. Delay before the hepatocyte phase of Gd-EOB-DTPA-enhanced MR imaging: is it possible to shorten the examination time? Eur Radiol. 2009; 19:26239.

22. Choi JS, Kim MJ, Choi JY, et al. Diffusion-weighted MR imaging of liver on 3.0-Tesla system: effect of intravenous administration of gadoxetic acid disodium. Eur Radiol. 2010;20:1052-60.

23. Huppertz A, Haraida S, Kraus A, et al. Enhancement of focal liver lesions at gadoxetic acid-enhanced MR imaging: correlation with histopathologic findings and spiral CT - initial observations. Radiology. 2005;234:468-78.

24. Zech CJ, Grazioli L, Breuer J, et al. Diagnostic performance and description of morphological features of focal nodular hyperplasia in Gd-EOB-DTPA-enhanced liver magnetic resonance imaging: results of a multicenter trial. Invest Radiol. 2008;43:504-11.

25. Purysko AS, Remer EM, Veniero JC. Focal liver lesion detection and characterization with GD-EOB-DTPA. Clin Radiol. 2011;66: 673-84.

26. Sano K, Ichikawa T, Motosugi U, et al. Imaging study of early hepatocellular carcinoma: usefulness of gadoxetic acid-enhanced MR imaging. Radiology. 2011;261:834-44.

27. Sun HY, Lee JM, Shin CI, et al. Gadoxetic acid-enhanced magnetic resonance imaging for differentiating small hepatocellular carcinomas ( $<$ or $=2 \mathrm{~cm}$ in diameter) from arterial enhancing pseudolesions: special emphasis on hepatobiliary phase imaging. Invest Radiol 2010;45:96-103.

28. Lee MH, Kim SH, Park MJ, et al. Gadoxetic acid-enhanced hepatobiliary phase MRI and high-b-value diffusion-weighted imaging to distinguish well-differentiated hepatocellular carcinomas from benign nodules in patients with chronic liver disease. AJR Am J Roentgenol. 2011;197:W868-75.

29. Kogita S, Imai Y, Okada M, et al. Gd-EOB-DTPA-enhanced magnetic resonance images of hepatocellular carcinoma: correlation with histological grading and portal blood flow. Eur Radiol. 2010;20: 2405-13.

30. Zech CJ, Herrmann KA, Reiser MF, et al. MR imaging in patients with suspected liver metastases: value of liver-specific contrast agent Gd-EOB-DTPA. Magn Reson Med Sci. 2007;6:43-52.

31. van Kessel CS, de Boer E, ten Kate FJ, et al. Focal nodular hyperplasia: hepatobiliary enhancement patterns on gadoxetic-acid contrast-enhanced MRI. Abdom Imaging. 2013;38:490-501.

32. Tiferes DA, D’Ippolito G. Neoplasias hepáticas: caracterização por métodos de imagem. Radiol Bras. 2008;41:119-27.

33. Denecke T, Steffen IG, Agarwal S, et al. Appearance of hepatocellular adenomas on gadoxetic acid-enhanced MRI. Eur Radiol. 2012;22:1769-75.

34. Ferlicot S, Kobeiter H, Tran Van Nhieu J, et al. MRI of atypical focal nodular hyperplasia of the liver: radiology-pathology correlation. AJR Am J Roentgenol. 2004;182:1227-31.

35. Bioulac-Sage P, Laumonier H, Couchy G, et al. Hepatocellular adenoma management and phenotypic classification: the Bordeaux experience. Hepatology. 2009;50:481-9.

36. Grazioli L, Bondioni MP, Haradome H, et al. Hepatocellular adenoma and focal nodular hyperplasia: value of gadoxetic acid-enhanced MR imaging in differential diagnosis. Radiology. 2012;262: 520-9.

37. Bartolozzi C, Battaglia V, Bozzi E. Hepatocellular nodules in liver cirrhosis: contrast-enhanced MR. Abdom Imaging. 201 1;36:290-9.

38. Bruix J, Sherman M, Llovet JM, et al. Clinical management of hepatocellular carcinoma. Conclusions of the Barcelona-2000 EASL conference. European Association for the Study of the Liver. J Hepatol. 2001;35:421-30.

39. Bruix J, Sherman M; Practice Guidelines Committee, American Association for the Study of Liver Diseases. Management of hepatocellular carcinoma. Hepatology. 2005;42:1208-36.

40. Narita M, Hatano E, Arizono S, et al. Expression of OATP1B3 determines uptake of Gd-EOB-DTPA in hepatocellular carcinoma. J Gastroenterol. 2009;44:793-8.

41. Zech CJ, Reiser MF, Herrmann KA. Imaging of hepatocellular carcinoma by computed tomography and magnetic resonance imaging: state of the art. Dig Dis. 2009;27:114-24.

42. Kim YK, Kim CS, Han YM, et al. Detection of hepatocellular carcinoma: gadoxetic acid-enhanced 3-dimensional magnetic resonance imaging versus multi-detector row computed tomography. J Comput Assist Tomogr. 2009;33:844-50.

43. Di Martino M, Marin D, Guerrisi A, et al. Intraindividual comparison of gadoxetate disodium-enhanced MR imaging and 64-section multidetector CT in the detection of hepatocellular carcinoma in patients with cirrhosis. Radiology. 2010;256:806-16.

44. Kim SH, Lee J, Kim MJ, et al. Gadoxetic acid-enhanced MRI versus triple-phase MDCT for the preoperative detection of hepatocellular carcinoma. AJR Am J Roentgenol. 2009;192:1675-81.

45. Chou CT, Chen YL, Su WW, et al. Characterization of cirrhotic nodules with gadoxetic acid-enhanced magnetic resonance imaging: the efficacy of hepatocyte-phase imaging. J Magn Reson Imaging. 2010;32:895-902.

46. Ahn SS, Kim MJ, Lim JS, et al. Added value of gadoxetic acid-enhanced hepatobiliary phase MR imaging in the diagnosis of hepatocellular carcinoma. Radiology. 2010;255:459-66.

47. Chan VO, Das JP, Gerstenmaier JF, et al. Diagnostic performance of MDCT, PET/CT and gadoxetic acid (Primovist $\left({ }^{\circledR}\right)$ )-enhanced MRI in patients with colorectal liver metastases being considered for hepatic resection: initial experience in a single centre. Ir J Med Sci. 2012;181:499-509.

48. Lee NK, Kim S, Lee JW, et al. Biliary MR imaging with Gd-EOBDTPA and its clinical applications. Radiographics. 2009;29:170724. 